\title{
INTERAÇÕES MEDICAMENTOSAS MAIS COMUNS COM OS ANTI- HIPERTENSIVOS: UMA REVISÃO DE LITERATURA
}

\section{ARTIGO DE REVISÃO}

MENDES, Driely Dandary Soares ${ }^{1}$

LOPES, Yanna Gabriele Guimarães ${ }^{2}$

DUARTE, Gabriella Mendes ${ }^{3}$

MENDES, Driely Dandary Soares. LOPES, Yanna Gabriele Guimarães. DUARTE, Gabriella Mendes. Interações medicamentosas mais comuns com os antihipertensivos: Uma revisão de literatura. Revista Científica Multidisciplinar Núcleo do Conhecimento. Ano 05, Ed. 11, Vol. 17, pp. 103-113. Novembro de 2020. ISSN: 2448-0959, Link de acesso: https://www.nucleodoconhecimento.com.br/saude/antihipertensivos

\section{RESUMO}

Introdução: A hipertensão Arterial é umas das doenças mais prevalentes mundialmente, associada a complicações cardiovasculares. Dessa forma mostra-se a importância de reduzir os níveis pressóricos, para isso os pacientes fazem uso de associações medicamentosas e essa necessidade para tratar a hipertensão e outra comorbidades pode causar interações medicamentosas, afetando diretamente na efetividade do tratamento farmacológico. Com isso, o presente artigo teve como

1 Graduanda em Farmácia na Universidade Potiguar, Laureate International Universities - Mossoró/Brasil.

2 Graduanda em Farmácia na Universidade Potiguar, Laureate International Universities - Mossoró/Brasil.

${ }^{3}$ Orientadora. Pós-graduada em Farmácia Clínica e Prescrição Farmacêutica pelo Instituto de Ciência, Tecnologia e Qualidade - Natal/Brasil. 
objetivo realizar uma revisão bibliográfica para identificar as interações mais comuns com medicamentos anti-hipertensivos. Metodologia: Trata-se de uma revisão integrativa da literatura pela análise de pesquisas sobre o assunto demostrados em estudos quantitativos. A busca dos artigos se deu por bases de dados Scielo, Medline, Pubmed, Periódicos Capes e Biblioteca Virtual em Saúde, entre o período de 2000 a 2020, visando selecionar trabalhos que tiveram grande impacto social e construir uma base de dados consistente. Resultados e Discussão: Diante da revisão bibliográfica foi possível verificar a presença de hipertensão arterial em indivíduos com faixa etária entre 55 anos a 72 anos, sendo mais presente no gênero feminino, e entre as interações medicamentosas com anti-hipertensivos, as mais prevalentes foram seis interações de acordo com as classes terapêuticas, que são: Diuréticos e Inibidores da Enzima Conversora Angiotensina; Bloqueadores dos Receptores de Angiotensina e Antiinflamatórios Não Esteroidais; Antiinflamatórios Não Esteroidais e Diuréticos; Inibidores da Enzima Conversora de Angiotensina e Bloqueadores dos Receptores de Angiotensina; Antiinflamatórios Não Esteroidais e Inibidores da Enzima Conversora de Angiotensina e Beta Bloqueadores e Antiinflamatórios Não Esteroidais. Conclusão: No presente artigo direcionado aos estudos dos anti-hipertensivos, pode-se verificar as prevalências de interações comumente prescritas aos pacientes e seus possíveis prejuízos à saúde, afetando diretamente a efetividade da farmacoterapia do paciente, ocasionando um problema de saúde que requer intervenções de profissionais, principalmente do farmacêutico que se destaca como o profissional do tratamento e o meio de reverter prejuízos causados por medicamentos, melhorando a qualidade de vida e promovendo a saúde.

Palavras Chaves: Interações medicamentosas, Hipertensão Arterial, AntiHipertensivos, Atenção Farmacêutica.

\section{INTRODUÇÃO}

Segundo a Diretriz Brasileira de Hipertensão Arterial, a hipertensão arterial é uma condição clínica multifatorial, caracterizada pela elevação dos níveis pressóricos maiores ou igual a $140 \mathrm{mmHg}$ da pressão sistólica e maior ou igual a $90 \mathrm{mmHg}$ para pressão diastólica. Essa patologia estar associada a distúrbios metabólicos, 
alterações funcionais, sexo, etnia, idade, ingestão de sódio, álcool, excesso de peso, sedentarismo, fatores socioeconômicos e genéticos (MALACHIAS, 2016). No Brasil, a hipertensão arterial atinge cerca de $32,5 \%$ de indivíduos adultos, contribuindo para o aumento dos índices de complicações cardiovasculares. Em 2013 ocorreram 1.138.670 óbitos, dos quais $29,8 \%$ foram causadas por doenças cardiovasculares (MALACHIAS, 2016).

Em vista de a hipertensão ser umas das doenças mais prevalentes no mundo, o governo desenvolve políticas públicas de saúde para estimular o diagnóstico precoce e o tratamento contínuo para controle da pressão arterial e dos fatores de riscos associados por meio da mudança do estilo de vida e/ ou uso de medicamentos (MALACHIAS, 2016). Diante da dificuldade em manter as metas pressóricas, verificase que para abordagem terapêutica do paciente hipertenso é necessário que haja um tratamento não farmacológico concomitante com o tratamento farmacológico e muitas vezes necessitando de associação medicamentosa, por causa do aumento de doenças crônico-degenerativas (PINHEIRO; CARVALHO; LUPPI, 2013). Esse aumento de consumo de medicamentos tanto para tratar a hipertensão arterial como para tratar outras comorbidades, gera consequências da poli farmácia (uso simultâneo de vários medicamentos), o que aumenta as chances de interações medicamentosas podendo ocasionar problemas de saúde para o paciente, visto que grande parte dos medicamentos é metabolizada por vias enzimáticas do citocromo monoxidade P450 (CYP), uns dos principais mecanismos envolvidos na ocorrência de interações medicamentosas (CAMPANHA et al, 2009; CORRER, 2002; MALACHIAS, 2016; PINHEIRO; CARVALHO; LUPPI, 2013).

As interações medicamentosas podem gerar uma diminuição ou exacerbação da efetividade terapêutica, ocasionando problemas de insegurança e dificuldade na manutenção dos níveis pressóricos que, consequentemente, aumentam as doenças cardiovasculares (GOTIJO et al, 2012). Denota-se, então, um requerimento de atenção na terapia farmacológica da hipertensão arterial, principalmente por ser uma doença que atinge cerca de 2,8\% dos adultos com 18 a 29 anos, 20,6\% aos de 30 a 59 anos, $44,4 \%$ aos de 60 a 64 anos, $52,7 \%$ aos de 65 a 74 anos e $55 \%$ aos de mais 
de 75 anos (MALACHIAS, 2016). Assim, esse trabalho teve como objetivo revisar artigos já publicados e avaliar as evidências de estudos em relação aos principais fármacos utilizados no tratamento para hipertensão arterial e identificar a incidências das principais interações medicamentosas que podem ocasionar potenciais riscos aos pacientes hipertensos.

\section{METODOLOGIA}

Trata-se de uma revisão integrativa da literatura pela análise de pesquisas sobre o assunto demostrado em estudos quantitativos que dão suporte a prática clínica. Sendo o objetivo principal a síntese dos estudos sobre interações medicamentosas com drogas anti-hipertensivas, os problemas relacionados ao medicamento e a importância da atenção farmacêutica diante dos obstáculos para a não efetividade terapêutica.

A busca dos artigos se deu empregando-se os seguintes descritores em português: "hipertensão arterial", "interações medicamentosas", "interações medicamentosas em anti-hipertensivos", "prevalência de interações medicamentosas prescritas em pacientes hipertensos", "problemas relacionados aos medicamentos no contexto farmacêutico", através das bases de dados Scielo, Medline, Pubmed, Periodicos Carpes e Biblioteca Virtual em Saúde.

Os critérios de inclusão utilizados para a busca foram artigos publicados entre $2000 \mathrm{e}$ 2020, visando selecionar trabalhos que tiveram grande impacto social e construir uma base de dados consistente. Os critérios de exclusão incluíram relatórios técnicos, teses e artigos que não tratavam do tema exposto.

A seleção dos artigos foi guiada por meio dos títulos dos trabalhos e seus resumos para que fosse possível verificar a abrangência dos conteúdos dos mesmos. Em seguida, houve um mapeamento das produções de acordo com o tipo de estudo empregado, visando constatar o objetivo da presente revisão. 


\section{RESULTADOS E DISCUSSÃO}

Diante da revisão bibliográfica dos estudos quantitativos e qualitativos, foi possível verificar a presença de hipertensão arterial em indivíduos com faixa etária entre 55 anos a 72 anos, tendo em sua maioria mais presente no gênero feminino, como pode ser constatado pelo estudo transversal com 356 indivíduos, cujos participantes tinham idade igual ou superior a 18 anos e diagnóstico de hipertensão arterial, em que 75\% dos pacientes com hipertensão arterial eram mulheres (ANDRADE; SOUZA, 2018). Esse fato se deve, provavelmente, pelas mulheres buscarem com maior frequência o cuidado da saúde, sendo diagnosticadas mais precocemente.

As interações entre os fármacos e anti-hipertensivos foram selecionadas de acordo com as classes terapêuticas disponíveis na Diretriz da Hipertensão Arterial, em que foi verificada a prevalência de seis interações que são: Diuréticos e Inibidores da Enzima Conversora Angiotensina (IECA); Bloqueadores dos Receptores de Angiotensina e Antiinflamatórios Não Esteroidais; Antiinflamatórios Não Esteroidais e Diuréticos; Inibidores da Enzima Conversora de Angiotensina e Bloqueadores dos Receptores de Angiotensina; Antiinflamatórios Não Esteroidais e Inibidores da Enzima Conversora de Angiotensina e Beta Bloqueadores e Antiinflamatórios Não Esteroidais. Além disso, os medicamentos foram consultados de acordo a Relação Nacional de Medicamentos de 2017.

Para melhor compreensão das interações medicamentosas, foram apresentados os mecanismos de ação das classes terapêuticas das associações mais prevalentes nas interações medicamentosas, como pode ser visto pela Tabela 1.

Tabela 1: Mecanismos de ação das classes terapêuticas utilizadas por pacientes com HAS, mais presentes nas interações medicamentosas.

\section{CLASSE TERAPÊUTICA MECANISMO DE AÇÃO}

$\begin{array}{ll}\text { Diuréticos } & \begin{array}{l}\text { Diminuição do volume extracelular (MALACHIAS, } \\ \text { 2016). }\end{array}\end{array}$


Inibidores da Enzima Impede a transformação de angiotensina I em Conversora

de angiotensina II que tem ação vasoconstrição com Angiotensina (IECA) redução temporária da filtração glomerular (GONZAGA, JUNIOR, AMOEDO, 2009/ MALACHIAS, 2016).

Anti-inflamatórios Não Inibe a produção de prostaglandinas por meio da Esteroidais (AINE's) competição com o sítio ativo da enzima ciclogenase (COX) (NASCIMENTO, PIGOSO, 2013).

Bloqueador do Receptor Antagonizam a ação da angiotensina II por meio do de Angiotensina (BRA) bloqueio específico dos receptores $A T_{1}$, que são responsáveis pela ação provocada pela angiotensina II, ações estimuladoras de liberação de aldosterona e vasoconstritoras (MALACHIAS, 2016).

Betabloqueadores Diminui o débito cardíaco e da secreção de renina e diminuição da ação do sistema nervoso pela readaptação dos barroceprotes (MALACHIAS, 2016).

Fonte: Mendes; Lopes; Duarte, 2020.

\subsection{INTERAÇÃO ENTRE DIURÉTICOS E INIBIDORES DA ENZIMA CONVERSORA DE ANGIOTENSINA}

Entre os representantes dos IECAs, o mais comumente citado foi o captopril e entre os diuréticos foram a hidroclorotiazida, espironolactona e furosemida. A interação entre captopril e hidroclorotiazida chegou a ter cerca de $44 \%$ entre as interações prevalentes no estudo transversal realizado em pacientes em uso de fármacos antihipertensivos (ANDRADE; SOUZA, 2018). Esse tipo de associação em dose baixa oferece vantagens como a de melhorar o controle da pressão arterial, proteção cardíaca e baixa incidência de efeitos colaterais [11], porém se administrados de forma incorreta podem levar a hipotensão postural por vasodilatação ou comprometimento da pressão de perfusão renal e filtração glomerular (ANDRADE; SOUZA, 2018; MONTEIRO et al, 2015). 
A associação entre o diurético poupador de potássio - espironolactona e captopril pode induzir hipercalemia em pacientes de risco, diante da retenção de potássio e aumento de risco de arritmias (ANDRADE; SOUZA, 2018; GONZAGA; JUNIOR; AMOEDO, 2009), correspondendo cerca de $5 \%$ das interações medicamentosas potenciais em um estudo com medicamentos usados por uma amostra de pessoas de 60 anos ou mais, residentes no Rio de Janeiro (MIBIELLI; ROZENFED; ACURCIO, 2014).

A utilização da furosemida aumenta-se o risco de insuficiência renal ao inibirem a aldosterona, hipotensão e hiponatremia, citado com o segundo mais prevalente no estudo dos principais fármacos utilizados no tratamento de hipertensão, no Centro de Saúde de Rincão - SP, correspondendo cerca de $12 \%$ das interações (PINHEIRO; CARVALHO; LUPPI, 2013; SANTOS; SENGER, 2019; VERONEZ; SIMÕES, 2008).

Pode verifica-se que associação dos IECAs com diuréticos causa um efeito sinérgico anti-hipertensivo mais intenso e assim causando uma maior depleção de sódio devido a ativação do sistema renina-angiotensina-aldosterona (GONZAGA; JUNIOR; AMOEDO, 2009).

\subsection{INTERAÇÃO ENTRE OS BLOQUEADORES DOS RECEPTORES DE ANGIOTENSINA E ANTI-INFLAMATÓRIOS NÃO ESTEROIDAIS}

Entre os medicamentos dessa interação, os mais relatados foram a losartana (BRA) e o ácido acetilsalicílico (AINE's). Os anti-inflamatórios não esteroidais podem elevar os níveis pressóricos, devido à inibição da síntese de prostaglandinas renais que são responsáveis pelo aumento do fluxo sanguíneo renal e consequentemente ocorre a retenção de líquidos [11]. Porém, presume-se que o efeito benéfico relacionado à prevenção de doenças cardiovasculares, supere a possível elevação pressórica, inclusive em pacientes com hipertensão arterial, que se utiliza a associação em curto prazo 1 (ANDRADE; SOUZA, 2018; MONTEIRO et al, 2015).

Dessa forma, em casos que é necessário o uso da associação em uso crônico, pode estar sendo utilizada diante do uso de baixas doses (80 a 200mg) do ácido 
acetilsalicílico e com o devido acompanhamento para que haja monitoração da função renal, risco de hemorragia gastrointestinal e da pressão arterial (MONTEIRO et al, 2015).

Essa interação foi citada como responsável por cerca de $36 \%$ de frequência entre as interações potenciais (IMP), em um estudo descritivo, quantitativo, de corte transversal, desenvolvido em duas Unidades de Saúde da Família (USF), no município de São Francisco do Conde, com 356 participantes com idade igual ou superior a 18 anos cadastrados no Sistema de Cadastramento e Acompanhamento de Hipertensos e Diabéticos (ANDRADE; SOUZA, 2018).

\subsection{INTERAÇÃO ENTRE ANTI-INFLAMATÓRIOS NÃO ESTEROIDAIS E DIURÉTICOS}

As associações entre medicamentos anti-hipertensivos e AINE's podem interferir diretamente na qualidade de vida do paciente (SANTOS; JUNIOR; RESTINI, 2012). Nesse contexto, enquadram-se os diuréticos (furosemida, espirolactona, hidroclorotiazida, etc.) que possuem sua atividade afetada diretamente pelo os AINE's, principalmente pelo ácido acetilsalicílico que antagoniza o efeito terapêutico do diurético na secreção de sódio e dessa forma pode-se afetar a atividade da renina plasmática, potencializando a hipertensão do indivíduo (NASCIMENTO; PIGOSO, 2013).

Citada como uma das mais frequentes interações entre anti-hipertensivos e AINE's no estudo de avaliação de 600 pacientes com prescrições de anti-hipertensivos levados a farmácia comunitária do centro médico em Pradópolis, SP, apresentando cerca de 12\% das interações (SANTOS; JUNIOR; RESTINI, 2012). 


\subsection{INTERAÇÕES ENTRE INIBIDORES DA ENZIMA CONVERSORA DE ANGIOTENSINA (IECA) E BLOQUEADORES DOS RECEPTORES DE ANGIOTENSINA (BRA)}

Evidenciada como umas das potenciais interações medicamentosas graves podem ser comprovadas no estudo observacional de corte transversal com 257 pacientes hipertensos cadastrados e acompanhados no Programa do Sistema de Cadastramento e Acompanhamento de Hipertensos e Diabéticos (SIS-HIPERDIA), vinculada a uma Unidade Básica de Saúde (UBS), em São Luís, Maranhão (MONTEIRO et al, 2015).

A interação apresenta interferência na farmacodinâmica, diante do sinergismo dos fármacos, visto que as duas classes terapêutica atuam no sistema renina angiotensina (SANTOS; SENGER, 2019). Entre seus principais representantes, há losartana (BRA) e enalapril (IECA) que inibem a ações de vasoconstrição provocada pelo sistema renina-angiotensina-aldosterona (MONTEIRO et al, 2015). Dessa forma, essa associação pode elevar os riscos de hipotensão, disfunção renal e hipercalemia, que sendo de forma severa pode levar a insuficiência renal, paralisia muscular e complicações no ritmo cardíaco (MONTEIRO et al, 2015).

\subsection{INTERAÇÕES ENTRE ANTI-INFLAMATÓRIOS NÃO ESTEROIDES} (AINE'S) E INIBIDORES DA ENZIMA CONVERSORA DE ANGIOTENSINA (IECA)

A ocorrência dessa interação é comum, visto que o ácido acetilsalicílico é frequentemente utilizado em pacientes hipertensos, como antiagregante plaquetário para inibir eventos cardiovasculares. Essa interação é vista como relevante nos estudos, já que os IECA, principalmente o enalapril e captopril, aumentam a produção de prostaglandinas renais com ação vasodilatadora, o que pode ser antagonizado pelo uso de AINES diante da inibição da enzima cicloxigenase (MIBIELLI et al, 2014) Dessa forma, pode resultar na diminuição significativa da função renal e hipercalemia (SANTOS; SENGER, 2019 ). 
Há controvérsias em relação a essa associação, devido ao benefício da aspirina em evitar coágulos, em virtude disso, caso for necessário o uso prolongado dessa associação é necessário uso de doses baixas do AAS e monitoramento da função renal e da pressão arterial (GONZAGA; JUNIOR; AMOEDO, 2009).

Pode-se verificar a prevalência dessas interações em um estudo de avaliação de 600 pacientes com prescrições de anti-hipertensivos levados a farmácia comunitária do centro médico em Pradópolis, SP, em que a interação em questão foi citada como a segunda mais frequente (SANTOS; JUNIOR; RESTINI, 2012).

\subsection{INTERAÇÕES ENTRE BETAS BLOQUEADORES (BB) E ANTI- INFLAMATÓRIOS NÃO ESTEROIDAIS (AINES)}

Dentro dessa classe, temos como principais representantes dos Betas Bloqueadores o atenolol e propranolol e dos Anti-inflamatórios Não Esteroidais temos o ácido acetilsalicílico, uma associação presente como, por exemplo, em cerca de $14 \%$ das interações no estudo realizado em Rincão, SP, em 725 pacientes hipertensos cadastrados no Centro Municipal de Saúde que faziam acompanhamento trimestral (VERONEZ; SIMÕES, 2008).

Essa combinação pode atenuar os efeitos anti-hipertensivos dos betas bloqueadores diante da inibição das prostaglandinas renais pelo ácido acetilsalićlico e consequentemente prejuízo ao controle da pressão arterial do paciente hipertenso (MIBIELLI et al, 2014).

\section{CONCLUSÃO}

Diante das interações mais prevalentes nos artigos estudados, é possível verificar que os Anti-inflamatórios Não Esteroidais estão em maior frequência nas associações, demostrando que o uso de AINES aumenta a pressão arterial por meio de diferentes mecanismos, por exemplo, a inibição da vasodilatação de prostaglandinas e aumento da reabsorção de sódio (PÓVOA, 2013; SOUZA; PIMENTA; BORELLI, 2009). 
Os AINES seletivos da Cox-2 foram sintetizados com a possibilidade de reduzir os efeitos colaterais atribuídos à inibição da Cox-1, porém, frequentemente causam problemas mais graves em associação indiscriminada com anti-hipertensivos, acarretando em complicações cardiovascular e renal (VERONEZ; SIMÕES, 2008). É possível verificar, também, que alguns estudos mostram que os empregos dessas duas classes podem ser benéficos diante do uso adequado, por exemplo, a utilização de doses de $2 \mathrm{mg}$ do ácido acetilsalićlico, devido sua ação antiagregante plaquetária. (OLIVEIRA et al, 2018).

No presente artigo direcionado aos hipertensos, pode-se verificar as prevalências de interações comumente prescritas aos pacientes e seus possíveis prejuízos à saúde, afetando diretamente a efetividade da farmacoterapia do paciente, ocasionando um problema de saúde que requer intervenções de profissionais (REINHARDT et al, 2012).

Dessa maneira, verifica-se que a maior parte dos indivíduos hipertensos são pessoas idosas e que fazem uso de poli farmácia devido às numerosas enfermidades, mostrando a necessidade de um monitoramento constante, não só da administração dos medicamentos, como também dos problemas relacionados à farmacoterapia e possíveis reações adversas (MILLHER et al, 2016; OPARIL, 2003).

Portanto, destaca-se a atenção farmacêutica, onde o farmacêutico diante de suas habilidades e raciocínio clínico se responsabiliza pelos problemas de saúde do paciente e identifica-os, a fim de desenvolver um plano terapêutico através de intervenções, como reduzir o sistema terapêutico, identificar as interações medicamentosas e erros de medicação, promover a adesão terapêutica e a educação em relação aos medicamentos. Logo, o farmacêutico destaca-se como o profissional no tratamento, responsável por reverter prejuízos causados por medicamentos, melhorando a qualidade de vida e promovendo a saúde (FEDRECICO, 2012; REINHARDT et al, 2012). 


\section{REFERÊNCIAS}

ANDRADE, Kaio Vinicius Freitas; SOUZA, Alyne Mascarenhas. Prevalência de interações medicamentosas potenciais em indivíduos hipertensos acompanhados na estratégia de saúde da família. J.Health Biol Sci. v. 6, n.4. p. 405-411. Agosto, 2018.

CAMPANA, Érika Maria Gonçalves; LEMOS, Christiano Canellas; MAGALHÃES, Maria Eliane Campos; BRANDÃO, Andréa Araújo. Interações e associações medicamentosas no tratamento da hipertensão - Bloqueadores alfa-adrenérgicos e vasodilatadores diretos. Revista Brasileira de Hipertensão. v.16, n.4, p.231-236. Outubro/Dezembro, 2009.

CORRER, Cassyano Janúario. Os problemas relacionados aos medicamentos no contexto da atenção farmacêutica: uma avaliação de conceitos. Infarma. v.14, n5/6, p.73-79, 2002.

FREDERICO, Pablo Mibielli. Interações medicamentosas potenciais dos antihipertensivos: uso perigoso entre idosos. Escola Nacional de Saúde Pública Sergio Arouca. V.1, n.1, p.1-116. Março, 2012.

GONTIJO, Mônica de Fátima; RIBEIRO, Andréia Queiroz; KLEIN, Carlos Henrique; ROZENFELD, Suely; ACURCIO, Francisco de Assis. Uso de anti-hipertensivos e antidiabéticos por idosos: inquérito em Belo Horizonte, Minas Gerais, Brasil. Cadastro Saúde Pública, Rio de Janeiro. v. 28, n.7, p.1337-1346. Julho, 2012.

GONZAGA, Carolina C. JUNIOR, Oswaldo Passarelli ; AMOEDO, Celso . Interações medicamentosas: inibidores da enzima conversora da angiotensina, bloqueadores dos receptores da angiotensina II, inibidores diretos da renina. Revista Brasileira de Hipertensão. v.16, n.4, p.221-225. Outubro/Dezembro, 2009.

MALACHIAS, MVB; SOUZA, WKSB; PLAVNIK, FL; RODRIGUES, CIS; BRANDÃO, AA; NEVES, MFT. $7^{\text {a }}$ Diretriz Brasileira de Hipertensão Arterial. Arquivo Brasileiro Cardiologia 2016; v.107. n.3 , Supl.3. p.1-83. Setembro, 2016. 
MIBIELLI, Pablo; ROZENFED, Suely; MATOS, Guacira Corrêa; ACURCIO, Francisco de Assis. Interações medicamentosas potenciais entre idosos em uso dos antihipertensivos da Relação Nacional de Medicamentos Essenciais do Ministério da Saúde do Brasil. Cad. Saúde Pública, Rio de Janeiro. v.30, n.9, p.1947-1956. Setembro, 2014.

MILLHER, Jessica Cheistiny; RODRIGUES, Nikyallan Soares; RIBEIRO Natalha Felisbino; BARRETO, Juliano Gomes; OLIVEIRA, Cristiano Guilherme Alvez. Atenção Farmacêutica aos idosos hipertensos: Um estudo de caso do município de Aperibé, RJ. Acta Biomedicina Brasilensia. v.7, n.1, p.1-10. Julho, 2016.

MONTEIRO, Sally Cristina Moutinho; BELFORT, Ilka Kassandra Perreira; SOUZA, Wandson Rodrigues; BARROS, Clemilson da Silva: CAMPOS, Karla Valéria Santos. Estudo de potenciais interações medicamentosas em pacientes hipertensos. Infarma Ciencias Farmacêuticas. V.27, n.2, p.117-125. Abril, 2015.

NASCIMENTO, Daniela Martins; PIGOSO, Acácio Antônio. Interações medicamentosas entre anti-hipertensivos e Anti-inflamatórios Não Esteroidais. Revista Científica da FHO/UIARARAS. V.1, n.1, p.14-17, 2013.

OLIVEIRA, Ludmilla Maria; ARAÚJO, Guilherme Barbosa de Souza; FERREIRA, Jorge Otávio Gonçalves; FERRAS, Vinicius Gonçalves; CARVALHO, Carlos Rubens Ponchini; SILVA, Denise. Resposta de pacientes hipertensos sob tratamento medicamentoso de acordo com os níveis pressóricos. Acta Biomedicina Brasilensia. v.9, n.3, p.61-71. Dezembro, 2018.

OPARIL, Suzanne. Antihypertensive and Lipid-Lowering Treatment to Prevent Heart Attack Trial (ALLHAT). Hypertension. v.41, n.5, p.1006-1009, 2003.

PINHEIRO, Juliana Souza; CARVALHO, Maristela Ferreira Catão; LUPPI, Graziela. Interações Medicamentosas e a farmacoterapia de pacientes geriátricos com Síndromes demenciais. Revista Brasileira Geriatriaca e Gerontologia. V.16, n.4, p.303-314, 2013. 
PÓVOA Rui. I Posicionamento Brasileiro sobre Combinação de Fármacos Anti Hipertensivos. Arquivo Brasileiro de Cardiologia. V.102, n.3. p. 203 - 210. Dez, 2013.

REINHARDT, Fernanda; ZIULKOSKI, Ana Luiza; ANDRIGHETTI, Letícia Hoerbe; PERASSOLO, Magda Susana. Acompanhamento. farmacoterapêutico em idosos hipertensos residentes em um lar geriátrico, localizado na Região do Vale dos Sinos, Rio Grande do Sul, Brasil. Rev. Bras. Geriatr. Gerontol. V.15, n.1, p.109-117, 2012.

Relação Nacional de Medicamentos Essenciais: RENAME 2017 / Ministério da Saúde, Secretaria de Ciência, Tecnologia e Insumos Estratégicos, Departamento de Assistência Farmacêutica e Insumos Estratégicos. - Brasília: Ministério da Saúde, 2017. $210 \mathrm{p}$.

SANTOS, Andressa Rodrigues, SENGER, Franciane Rios. Avaliação da terapia medicamentosa de pacientes idosos portadores de hipertensão arterial sistêmica e diabetes mellitus tipo 2 atendidos em uma unidade básica de saúde no município de Xanxerê - SC. Rev. Epidemiol. Controle Infecç. Santa Cruz. V.9, n.2, p.155-160. Junho, 2019.

SANTOS, Júlio César; JUNIOR, Milton Faria; RESTINI, Carolina Baraldi Araujo. Potenciais interações medicamentosas identificadas em prescrições a pacientes hipertensos. Revista Brasileira Clinica Médica. v.10, n.4, p.308-317. Julho/Agosto, 2012.

SOUZA, Márcio Gonçalves; PIMENTA, Eduardo de Sousa; BORELLI, Flávio Antonio Oliveira. Interações e associações medicamentosas no tratamento da hipertensão Combinações fixas. Revista Brasileira de Hipertensão. v.16, n.4, p.237-241, Outubro/Dezembro, 2009.

VERONEZ, L. L; SIMÕES, M.J.S. Análise da prescrição de medicamentos de pacientes hipertensos atendidos pelo SUS da rede Municipal de Saúde de RincãoSp. Rev. Ciênc. Farm. Básica Apl. v. 29, n.1, p. 45-51. Julho, 2008. 
Enviado: Agosto, 2020.

Aprovado: Novembro, 2020. 